Iranian Journal of Mathematical Sciences and Informatics

Vol. 15, No. 1 (2020), pp 125-133

DOI: 10.21859/IJMSI.15.1.125

\title{
On Identities with Additive Mappings in Rings
}

\author{
Abu Zaid Ansari \\ Department of Mathematics, Faculty of Science, Islamic University of \\ Madinah, K.S.A \\ E-mail: ansari.abuzaid@gmail.com

\begin{abstract}
If $F, D: R \rightarrow R$ are additive mappings which satisfy $F\left(x^{n} y^{n}\right)=x^{n} F\left(y^{n}\right)+y^{n} D\left(x^{n}\right)$ for all $x, y \in R$. Then, $F$ is a generalized left derivation with associated Jordan left derivation $D$ on $R$. Similar type of result has been done for the other identity forcing to generalized derivation and at last an example has given in support of the theorems.
\end{abstract}

Keywords: Prime (Semiprime) ring, Additive mappings, Generalized (Jordan) left derivations, Generalized (Jordan) derivations, (Jordan)centralizers.

2010 Mathematics Subject Classification: 16D90, 16W25, 16N60

\section{INTRODUCTION}

In this paper $R$ is an associative ring with identity. A ring $R$ is $n$-torsion free, where $n>1$ is an integer, in case $n x=0, x \in R$ implies $x=0$. A ring $R$ is prime if $a R b=\{0\}$ implies $a=0$ or $b=0$, and is semiprime if $a R a=\{0\}$ implies $a=0$. An additive mapping $d: R \rightarrow R$ is called a derivation if $d(x y)=d(x) y+x d(y)$ holds for all pairs $x, y \in R$ and is called a Jordan derivation in case $d\left(x^{2}\right)=d(x) x+x d(x)$ is fulfilled for all $x \in R$. An additive mapping $f: R \rightarrow R$ is said to be a generalized derivation if there exists a derivation $d: R \rightarrow R$ such that $f(x y)=f(x) y+x d(y)$ for all $x, y \in R$. An additive mapping $D: R \rightarrow R$ is said to be a left derivation (resp. Jordan left derivation) if $D(x y)=x D(y)+y D(x)$ (resp. $D\left(x^{2}\right)=2 x D(x)$ ) holds for all $x, y \in R$. An additive mapping $D: R \rightarrow R$ is said to be a right derivation (resp.

Received 22 March 2017; Accepted 26 Jun 2017

(C)2020 Academic Center for Education, Culture and Research TMU 
Jordan right derivation) if $D(x y)=D(x) y+D(y) x\left(\right.$ resp. $\left.D\left(x^{2}\right)=2 D(x) x\right)$ holds for all $x, y \in R$. If $D$ is both left as well as right derivation, then $D$ is a derivation. Clearly, every left (resp. right) derivation on a ring $R$ is a Jordan left (resp. Jordan right) derivation but the converse need not be true in general. Following Zalar [14], an additive mapping $T: R \rightarrow R$ is called left (resp. right) centralizer of $R$ if $T(x y)=T(x) y$ (resp. $T(x y)=x T(y)$ ) for all $x, y \in R$. In particular $T$ is Jordan left (resp. Jordan right) centralizer of $R$ if $x=y$. Obviously, every centralizer is a Jordan centralizer on $R$ but the converse is not true in general. Zalar in [14], proved: Every Jordan left centralizer on a 2-torsion free semiprime ring is a left centralizer. Following Ashraf et. al. [3], an additive mapping $F: R \rightarrow R$ is said to be a generalized left derivation (resp. generalized Jordan left derivation) if there exists a Jordan left deviation $D: R \rightarrow R$ such that $F(x y)=x F(y)+y D(x)$ ( resp. $F\left(x^{2}\right)=$ $x F(x)+x D(x))$ for all $x, y \in R$. $F$ is a generalized left derivation if and only if $F$ is of the form $F=T+D$, where $T$ right centralizer of $R$ and $D$ is a left derivation. The concept of generalized left derivations cover the concept of left derivation and if $D=0, F$ includes the concept of right centralizer. In 2013 [4], Ashraf et. al had proved that additive mappings $F, D: R \rightarrow R$ satisfying the properties $F\left(x^{n+1}\right)=x^{n} F(x)+n x^{n} D(x)$ for all $x \in R$, and show that if $R$ is a $(n+1)$ !-torsion free ring with identity, then $D$ is Jordan left derivation and $F$ is generalized Jordan left derivation on $R$. Similar type of result has been done in $[2,5]$. In view of $[2,4,5]$, we extend the results in the following setting.

\section{Main Results}

Theorem 2.1. Let $n>1$ be a fixed integer and $R$ be any $n$-torsion free ring. If $F, D: R \rightarrow R$ are additive mappings satisfying $F\left(x^{n} y^{n}\right)=x^{n} F\left(y^{n}\right)+y^{n} D\left(x^{n}\right)$ for all $x, y \in R$. Then, $F$ is generalized left derivation with associated Jordan left derivation $D$ on $R$.

Proof. We have

$$
F\left(x^{n} y^{n}\right)=x^{n} F\left(y^{n}\right)+y^{n} D\left(x^{n}\right) \text { for all } x, y \in R \text {. }
$$

Replacing $x$ by $e$ in the above equation, we get $D(e)=0$. Again, replace $x$ by $x+e$ in the above equation to get 


$$
\begin{aligned}
\left(\begin{array}{c}
n \\
0
\end{array}\right)\left[F\left(x^{n} y^{n}\right)\right. & \left.-x^{n} F\left(y^{n}\right)-y^{n} D\left(x^{n}\right)\right]+\left(\begin{array}{c}
n \\
1
\end{array}\right)\left[F\left(x^{n-1} y^{n}\right)-x^{n-1} F\left(y^{n}\right)\right. \\
& \left.-y^{n} D\left(x^{n-1}\right)\right]+\left(\begin{array}{c}
n \\
2
\end{array}\right)\left[F\left(x^{n-2} y^{n}\right)-x^{n-2} F\left(y^{n}\right)\right. \\
& \left.-y^{n} D\left(x^{n-2}\right)\right]+\ldots+\left(\begin{array}{c}
n \\
n-1
\end{array}\right)\left[F\left(x y^{n}\right)-x F\left(y^{n}\right)\right. \\
& \left.-y^{n} D(x)\right]+\left(\begin{array}{c}
n \\
n
\end{array}\right)\left[F\left(y^{n}\right)-F\left(y^{n}\right)-y^{n} D(e)\right]=0 .
\end{aligned}
$$

Using (2.1) together with the fact that $D(e)=0$, we have

$$
\begin{aligned}
\left(\begin{array}{c}
n \\
1
\end{array}\right)\left[F\left(x^{n-1} y^{n}\right)\right. & \left.-x^{n-1} F\left(y^{n}\right)-y^{n} D\left(x^{n-1}\right)\right]+\left(\begin{array}{c}
n \\
2
\end{array}\right)\left[F\left(x^{n-2} y^{n}\right)\right. \\
& \left.-x^{n-2} F\left(y^{n}\right)-y^{n} D\left(x^{n-2}\right)\right]+\ldots+\left(\begin{array}{c}
n \\
n-1
\end{array}\right)\left[F\left(x y^{n}\right)\right. \\
& \left.-x F\left(y^{n}\right)-y^{n} D(x)\right]=0 .
\end{aligned}
$$

Replacing $x$ by $k x$, we obtain

$$
\begin{aligned}
\left(\begin{array}{c}
n \\
1
\end{array}\right) k^{n-1}\left[F\left(x^{n-1} y^{n}\right)\right. & \left.-x^{n-1} F\left(y^{n}\right)-y^{n} D\left(x^{n-1}\right)\right]+\left(\begin{array}{c}
n \\
2
\end{array}\right) k^{n-2}\left[F\left(x^{n-2} y^{n}\right)\right. \\
& \left.-x^{n-2} F\left(y^{n}\right)-y^{n} D\left(x^{n-2}\right)\right] \ldots+\left(\begin{array}{c}
n \\
n-1
\end{array}\right) k\left[F\left(x y^{n}\right)\right. \\
& \left.-x F\left(y^{n}\right)-y^{n} D(x)\right]=0 .
\end{aligned}
$$

We can write the above equation as

$$
\sum_{r=1}^{n-1}\left(\begin{array}{c}
n \\
r
\end{array}\right) k^{n-r}\left[F\left(x^{n-r} y^{n}\right)-x^{n-r} F\left(y^{n}\right)-y^{n} D\left(x^{n-r}\right)\right]=0 \text { for all } x, y \in R .
$$

Replace $k$ by $1,2, \cdots, n-1$ in turn and consider the resulting system of $n-1$ homogeneous equations to get that the matrix of the system is a Van der Monde matrix

$$
\mathbb{V}=\left(\begin{array}{cccc}
1 & 1 & \cdots & 1 \\
2 & 2^{2} & \cdots & 2^{n} \\
\cdot & \cdot & \cdots & \cdot \\
\cdot & \cdot & \cdots & \cdot \\
\cdot & \cdot & \cdots & \cdot \\
n-1 & (n-1)^{2} & \cdots & (n-1)^{n}
\end{array}\right) .
$$

Here $|\mathbb{V}|=$ product of positive integer, each of which is less than $n-1$, it implies that

$$
\left(\begin{array}{c}
n \\
r
\end{array}\right) k^{n-r}\left[F\left(x^{n-r} y^{n}\right)-x^{n-r} F\left(y^{n}\right)-y^{n} D\left(x^{n-r}\right)\right]=0 \text { for all } x, y \in R
$$


$r=1,2, \cdots, n-1$. In particular take $r=n-1$, we obtain

$$
\left(\begin{array}{c}
n \\
n-1
\end{array}\right)\left[F\left(x y^{n}\right)-x F\left(y^{n}\right)-y^{n} D(x)\right]=0 \text { for all } x, y \in R .
$$

This yields

$$
n\left[F\left(x y^{n}\right)-x F\left(y^{n}\right)-y^{n} D(x)\right]=0 \text { for all } x, y \in R .
$$

Since $R$ is $n$-torsion free, we find that

$$
F\left(x y^{n}\right)=x F\left(y^{n}\right)+y^{n} D(x) \text { for all } x, y \in R .
$$

Again, replacing $y$ by $y+e$, we obtain

$$
\begin{aligned}
\left(\begin{array}{c}
n \\
0
\end{array}\right)\left[F\left(x y^{n}\right)\right. & \left.-x F\left(y^{n}\right)-y^{n} D(x)\right]+\left(\begin{array}{c}
n \\
1
\end{array}\right)\left[F\left(x y^{n-1}\right)-x F\left(y^{n-1}\right)\right. \\
& \left.-y^{n-1} D(x)\right]+\left(\begin{array}{c}
n \\
2
\end{array}\right)\left[F\left(x y^{n-2}\right)-x F\left(y^{n-2}\right)-y^{n-2} D(x)\right] \\
& +\ldots+\left(\begin{array}{c}
n \\
n-1
\end{array}\right)[F(x y)-x F(y)-y D(x)] \\
& +\left(\begin{array}{c}
n \\
n
\end{array}\right)[F(x)-x F(e)-D(x)]=0 .
\end{aligned}
$$

Taking $y=e$ in (2.2), we have

$$
F(x)=x F(e)+D(x) \text { for all } x \in R .
$$

Using (2.2) and (2.3) in the above relation, we have

$$
\begin{aligned}
\left(\begin{array}{c}
n \\
1
\end{array}\right)\left[F\left(x y^{n-1}\right)\right. & \left.-x F\left(y^{n-1}\right)-y^{n-1} D(x)\right] \\
& +\left(\begin{array}{c}
n \\
2
\end{array}\right)\left[F\left(x y^{n-2}\right)-x F\left(y^{n-2}\right)-y^{n-2} D(x)\right] \\
& +\ldots+\left(\begin{array}{c}
n \\
n-1
\end{array}\right)[F(x y)-x F(y)-y D(x)]=0 .
\end{aligned}
$$

Replacing $y$ by $k y$, we get

$$
\sum_{r=1}^{n-1}\left(\begin{array}{c}
n \\
r
\end{array}\right) k^{n-r}\left[F\left(x y^{n-r}\right)-x F\left(y^{n-r}\right)-y^{n-r} D(x)\right]=0 \text { for all } x, y \in R .
$$

Using the same steps as we did before equation (2.2), we arrive at

$$
F(x y)=x F(y)+y D(x) \text { for all } x, y \in R .
$$

Replace $x$ by $x^{2}$ in (2.3) to obtain $F\left(x^{2}\right)=x^{2} F(e)+D\left(x^{2}\right)$ for all $x \in R$. Again, replacing $y$ by $x$ in (2.4), we get $F\left(x^{2}\right)=x F(x)+x D(x)$ for all $x, y \in R$. Comparing the previous two relations, we get $x^{2} F(e)+D\left(x^{2}\right)=x F(x)+$ 
$x D(x))$ for all $x \in R$. This implies that $\left.D\left(x^{2}\right)=x(F(x)-x F(e))+x D(x)\right)$ for all $x \in R$. Again, using (2.3) in the previous relation, we get $D\left(x^{2}\right)=$ $2 x D(x)$ for all $x \in R$. Therefore, $D$ is a Jordan left derivation. Hence, $F$ is a generalized left derivation associated with $D$.

The Following corollary is a consequence of the above theorem:

Corollary 2.2. Let $n>1$ be a fixed integer and $R$ be any $n$-torsion free semiprime ring. If $F, D: R \rightarrow R$ are additive mappings satisfying $F\left(x^{n} y^{n}\right)=$ $x^{n} F\left(y^{n}\right)+y^{n} D\left(x^{n}\right)$ for all $x, y \in R$. Then

(1) $D$ is a derivation on $R$ and $[D(x), y]=0$ for all $x, y \in R$,

(2) $D$ is a derivation which maps $R$ into $Z(R)$,

(3) $R$ is commutative or $D=0$,

(4) $F(x)=x q$ for all $x \in R$ and some $q \in Q_{l}\left(R_{C}\right)$, where $Q_{l}\left(R_{C}\right)$ is left Martindale ring of quotients,

(5) $F$ is a generalized derivation on $R$.

Proof. (1) Given that $F\left(x^{n} y^{n}\right)=x^{n} F\left(y^{n}\right)+y^{n} D\left(x^{n}\right)$ for all $x, y \in R$. Then, by Theorem 2.1, $F$ is generalized left derivation and $D$ is Jordan left derivation. Hence, by [1, Theorem 3.1], $D$ is derivation and $[D(x), y]=0$ for all $x, y \in R$.

(2) Since $F\left(x^{n} y^{n}\right)=x^{n} F\left(y^{n}\right)+y^{n} D\left(x^{n}\right)$ for all $x, y \in R$. Then by Theorem 2.1, $F$ is generalized left derivation and $D$ is Jordan left derivation on $R$. Hence, by [13, Theorem 2], we get the required result.

(3) Assume that $D \neq 0$. We have $F\left(x^{n} y^{n}\right)=x^{n} F\left(y^{n}\right)+y^{n} D\left(x^{n}\right)$ for all $x, y \in R$. Then, by Theorem 2.1, $F$ is generalized left derivation and $D$ is Jordan left derivation. Hence, using (1) we find that $D$ is a derivation and $[D(x), y]=0$ for all $x, y \in R$. hence, in particular $[D(x), x]=0$ for all $x \in R$. Since $R$ is prime and $D$ is nonzero derivation, $R$ is commutative by [9, Theorem 2]

(4) We have $F\left(x^{n} y^{n}\right)=x^{n} F\left(y^{n}\right)+y^{n} D\left(x^{n}\right)$ for all $x, y \in R$. Thus by Theorem 2.1, $F$ is generalized left derivation on $R$. Since $R$ is a noncommutative 2-torsion free prime ring and $F$ is a generalized left derivation on $R$. In view of (3), we have $D=0$. Thus we obtain $F(x y)=x F(y)$ for all $x, y \in R$. That is $F$ is a right centralizer on $R$. Hence, there exists $q \in Q_{l}\left(R_{C}\right)$ such that $F(x)=x q$ for all $x \in R$ by Proposition 2.10 of $[1]$.

(5) Since $F\left(x^{n} y^{n}\right)=x^{n} F\left(y^{n}\right)+y^{n} D\left(x^{n}\right)$ for all $x, y \in R$. In view of Theorem 2.1 and (3), $D$ is a derivation and $R$ is commutative. Since $R$ is a 2-torsion free prime ring and $F$ is a generalized left derivation, we find that $F(y x)=F(x y)=x F(y)+y D(x)=F(y) x+x D(y)$ for all 
$x, y \in R$. This implies that $F(y x)=F(y) x+y D(x)$ for all $x, y \in R$. Hence, $F$ is generalized derivation on $R$.

Lemma $2.3([8])$. Any linear derivation on a semisimple Banach Algebra is continuous.

Lemma 2.4 ([10]). A continuous linear derivation on a commutative Banach Algebra maps algebra into its radical.

Combining the above two results, Thomos proved the following:

Lemma 2.5 ([11]). There does not exist any nonzero linear derivations on commutative semisimple Banach algebras.

In view of $[8,10,11]$, the following consequence has been given:

Theorem 2.6. Let $n>1$ be any fixed integer and $A$ be a semisimple Banach algebra and let $F, D: A \rightarrow A$ be additive mappings satisfying $F\left(x^{n} y^{n}\right)=$ $x^{n} F\left(y^{n}\right)+y^{n} D\left(x^{n}\right)$ for all $x, y \in A$. In this case $D=0$.

Proof. Since semisimple Banach algebra are semiprime, hence all the assumptions of Corollary 2.2 (1) are fulfilled. We have therefore a linear derivation on semisimple Banach algebra $A$. Hence, by Theorem 4 of [13], we get $D=0$.

Now, come to the next theorem.

Theorem 2.7. Let $n>1$ be a fixed integer and $R$ be any $n$-torsion free semiprime ring. If $f, d: R \rightarrow R$ are additive mappings satisfying $f\left(x^{n} y^{n}\right)=$ $f\left(x^{n}\right) y^{n}+x^{n} d\left(y^{n}\right)$ for all $x, y \in R$. Then, $f$ is generalized derivation with associated derivation $d$ on $R$.

Proof. We have

$$
f\left(x^{n} y^{n}\right)=f\left(x^{n}\right) y^{n}+x^{n} d\left(y^{n}\right) \text { for all } x, y \in R .
$$

Replacing $x$ by $e$ in the above equation, we get $d(e)=0$. Again, replacing $x$ by $x+e$ in (2.5), we get

$$
\begin{aligned}
\left(\begin{array}{c}
n \\
0
\end{array}\right)\left[f\left(x^{n} y^{n}\right)\right. & \left.-f\left(x^{n}\right) y^{n}-x^{n} d\left(y^{n}\right)\right]+\left(\begin{array}{c}
n \\
1
\end{array}\right)\left[f\left(x^{n-1} y^{n}\right)-f\left(x^{n-1}\right) y^{n}\right. \\
& \left.-x^{n-1} d\left(y^{n}\right)\right]+\left(\begin{array}{c}
n \\
2
\end{array}\right)\left[f\left(x^{n-2} y^{n}\right)-f\left(x^{n-2}\right) y^{n}\right. \\
& \left.-x^{n-2} d\left(y^{n}\right)\right]+\ldots+\left(\begin{array}{c}
n \\
n-1
\end{array}\right)\left[f\left(x y^{n}\right)-f(x) y^{n}-x d\left(y^{n}\right)\right] \\
& +\left(\begin{array}{c}
n \\
n
\end{array}\right)\left[f\left(y^{n}\right)-f(e) y^{n}-d\left(y^{n}\right)\right]=0
\end{aligned}
$$


Taking $x=e$ in (2.5), we get $f\left(y^{n}\right)=f(e) y^{n}+d\left(y^{n}\right)$ for all $x, y \in R$. Now using (2.5) together with the last relation, we have

$$
\begin{aligned}
&\left(\begin{array}{c}
n \\
1
\end{array}\right)\left[f\left(x^{n-1} y^{n}\right)\right.\left.-f\left(x^{n-1}\right) y^{n}-x^{n-1} d\left(y^{n}\right)\right] \\
&+\left(\begin{array}{c}
n \\
2
\end{array}\right)\left[f\left(x^{n-2} y^{n}\right)-f\left(x^{n-2}\right) y^{n}-x^{n-2} d\left(y^{n}\right)\right]+\ldots \\
&+\left(\begin{array}{c}
n \\
n-1
\end{array}\right)\left[f\left(x y^{n}\right)-f(x) y^{n}-x d\left(y^{n}\right)\right]=0 \\
& \sum_{r=1}^{n-1}\left(\begin{array}{c}
n \\
r
\end{array}\right) k^{n-r}\left[f\left(x^{n-r} y^{n}\right)-f\left(x^{n-r}\right) y^{n}-x^{n-r} d\left(y^{n}\right)\right]=0 \text { for all } x, y \in R .
\end{aligned}
$$

By the same logic the resulting matrix of the system is a Van der Monde matrix. Hence,

$$
\left(\begin{array}{c}
n \\
r
\end{array}\right) k^{n-r}\left[f\left(x^{n-r} y^{n}\right)-f\left(x^{n-r}\right) y^{n}-x^{n-r} d\left(y^{n}\right)\right]=0 \text { for all } x, y \in R,
$$

$r=1,2, \cdots, n-1$. Now, in particular take $r=n-1$ and the fact that $R$ is $n$-torsion free, we get

$$
f\left(x y^{n}\right)=f(x) y^{n}+x d\left(y^{n}\right) \text { for all } x, y \in R .
$$

Again replacing $y$ by $y+e$ and use the fact that $d(e)=0$, we obtain

$$
\begin{aligned}
\left(\begin{array}{c}
n \\
1
\end{array}\right)\left[f\left(x y^{n-1}\right)\right. & \left.-f(x) y^{n-1}-x d\left(y^{n-1}\right)\right]+\left(\begin{array}{c}
n \\
2
\end{array}\right)\left[f\left(x y^{n-2}\right)-f(x) y^{n-2}\right. \\
& \left.\left.-x d\left(y^{n-2}\right)\right]+\ldots+\left(\begin{array}{c}
n \\
n-1
\end{array}\right)[f(x y)-f(x) y-x d(y)]\right]=0 .
\end{aligned}
$$

Replace $y$ by $k y$ to get

$$
\sum_{r=1}^{n-1}\left(\begin{array}{c}
n \\
r
\end{array}\right) k^{n-r}\left[f\left(x y^{n-r}\right)-f(x) y^{n-r}-x d\left(y^{n-r}\right)\right]=0 \text { for all } x, y \in R .
$$

Use the same technique to obtain

$$
f(x y)=f(x) y+x d(y) \text { for all } x, y \in R .
$$

Replacing $y$ by $y z$ in the above relation, we obtain,

$$
f(x y z)=f(x) y z+x d(y z) \text { for all } x, y, z \in R .
$$

Using (2.7), we arrive at

$$
f(x y) z+x y d(z)=f(x) y z+x d(y z) \text { for all } x, y, z \in R .
$$


Again using (2.7), we obtain

$$
f(x) y z+x d(y) z+x y d(z)=f(x) y z+x d(y z) \text { for all } x, y, z \in R .
$$

On simplifying, we have

$$
x(d(y z)-d(y) z-y d(z))=0 \text { for all } x, y, z \in R .
$$

Multiplying both side by $d(y z)-d(y) z-y d(z)$, we find

$$
(d(y z)-d(y) z-y d(z) x(d(y z)-d(y) z-y d(z))=0 \text { for all } x, y, z \in R .
$$

Using semiprimeness, we conclude that $d(y z)=d(y) z+y d(z)$ for all $y, z \in R$. Hence $d$ is a derivation. Therefore $f$ is a generalized derivation on $R$.

Corollary 2.8. Let $n>1$ be a fixed integer and $R$ be any $n$-torsion free semiprime ring. If $F: R \rightarrow R$ are additive mappings satisfying $F\left(x^{n} y^{n}\right)=$ $F\left(x^{n}\right) y^{n}$ and $F\left(x^{n} y^{n}\right)=x^{n} F\left(y^{n}\right)$ for all $x, y \in R$. Then, $F$ is a centralizer on $R$.

Proof. Taking $D=d=0$ in Theorem 2.1 and 2.7, we get the required result.

The following example is in the favour of our theorems.

Example 2.9. Define $R=\left\{\left(\begin{array}{ll}a & 0 \\ 0 & b\end{array}\right) \mid a, b \in 2 \mathbb{Z}_{8}\right\}, \mathbb{Z}_{8}$ is the ring of addition and multiplication modulo 8. Define mappings $F, D, f, d: R \rightarrow$ $R$ by $F\left(\begin{array}{ll}a & 0 \\ 0 & b\end{array}\right)=\left(\begin{array}{ll}0 & 0 \\ 0 & b\end{array}\right), D\left(\begin{array}{ll}a & 0 \\ 0 & b\end{array}\right)=\left(\begin{array}{ll}a & 0 \\ 0 & 0\end{array}\right), f\left(\begin{array}{ll}a & 0 \\ 0 & b\end{array}\right)=$ $\left(\begin{array}{ll}0 & 0 \\ 0 & b\end{array}\right)$ and $d\left(\begin{array}{ll}a & 0 \\ 0 & b\end{array}\right)=\left(\begin{array}{ll}a & 0 \\ 0 & 0\end{array}\right)$. It is clear that $F$ is not a generalized left derivation and $f$ is not a generalized derivation on $R$ but $F, D, f, d$ satisfy the identities $F\left(x^{2} y^{2}\right)=x^{2} F\left(y^{2}\right)+y^{2} D\left(x^{2}\right)$ and $f\left(x^{2} y^{2}\right)=f\left(x^{2}\right) y^{2}+$ $x^{2} d\left(y^{2}\right)$ for all $x, y \in R$.

\section{ACKNOWLEDGMENTS}

This research is supported by the project number 84/40, Deanship of Scientific research, Islamic University of Madinah, KSA.

\section{REFERENCES}

1. S. Ali, On generalized left derivations in rings and Banach algebras, Aequat. Math., 81, (2011), 209-226.

2. A. Z. Ansari, F. Shujat, Additive mappings satisfying algebraic conditions in rings Rendiconti del Circolo Matematico di Palermo, 63(2), (2014), 211-219.

3. M. Ashraf, S. Ali, On generalized Jordan left derivations in rings, Bull. Korean Math. Soc. 45(2), (2008), 253-261. 
4. M. Ashraf, N. Rehman, and A. Z. Ansari, An additive mapping satisfying an algebraic condition in rings with identity, Journal of Advanced Research in Pure Mathematics, 5(2), (2013), 38-45.

5. B. Dhara, R. K. Sharma, On addtive mappings in rings with identity elements, Interenational Mathematical Forum, 4(15), 2009, 727-732.

6. I. N. Herstein, Derivations in prime rings, Proc. Amer. Math. Soc., 8, (1957), 1104-1110.

7. I. N. Herstein, Topics in ring theory, Univ. Chicago Press, Chicago, 1969.

8. B. E. Johnson, and A.M. Sinklair, Continuity of derivations and a problem of Kaplansky, Amer. J. Math., 90, (1968), 1068-1073.

9. E. C. Posner, Derivations in prime rings, Proc. amer. Math. Soc., (1957), 1093-1100.

10. I. M. Singer, and J. Wermer, Derivations on commutative normes spaces, Math. Ann., 129, (1995) 435-460.

11. M.P. Thomos, The image of a derivation is contained in the radical, Annals of Math., 128, (1988), 435-460.

12. Vukman, J. Jordan left derivations on semiprime rings, Math. J. Okayama Univ., 39, 1-6 (1997).

13. Vukman, J. On left Jordan derivations on rings and Banach algebras, Aequationes Math, 75, (2008), 260-266.

14. Zalar, B. On centralizers of semiprime rings, Comment. Math. Univ. carolin., 32(4), (1991) 609-614. 